DRAFT VERSION APRIL 30, 2019

Preprint typeset using LATEX style emulateapj v. 26/01/00

\title{
THE OUTER RIM SIMULATION: A PATH TO MANY-CORE SUPERCOMPUTERS
}

\author{
Katrin Heitmann ${ }^{1}$, Hal Finkel $^{2}$, Adrian Pope ${ }^{3}$, Vitali Morozov ${ }^{2}$, Nicholas Frontiere ${ }^{1,4}$, \\ Salman Habib ${ }^{1,3}$, Esteban Rangel ${ }^{2}$, Thomas URAM ${ }^{2}$, Danila Korytov ${ }^{1,4}$, Hillary ChILD ${ }^{1,4}$, \\ SAMUEL FLENDER ${ }^{2}$, JOE INSLEY ${ }^{2}$, SILVIO RIZZI ${ }^{2}$ \\ ${ }^{1}$ HEP Division, Argonne National Laboratory, Lemont, IL 60439 \\ 2 ALCF Division, Argonne National Laboratory, Lemont, IL 60439 \\ ${ }^{3}$ CPS Division, Argonne National Laboratory, Lemont, IL 60439 \\ ${ }^{4}$ Department of Physics, University of Chicago, Chicago, IL 60637
}

Draft version April 30, 2019

\begin{abstract}
We describe the Outer Rim cosmological simulation, one of the largest high-resolution N-body simulations performed to date, aimed at promoting science to be carried out with large-scale structure surveys. The simulation covers a volume of $(4.225 \mathrm{Gpc})^{3}$ and evolves more than one trillion particles. It was executed on Mira, a BlueGene/Q system at the Argonne Leadership Computing Facility. We discuss some of the computational challenges posed by a system like Mira, a many-core supercomputer, and how the simulation code, HACC, has been designed to overcome these challenges. We have carried out a large range of analyses on the simulation data and we report on the results as well as the data products that have been generated. The full data set generated by the simulation totals more than $5 \mathrm{~PB}$ of data, making data curation and data handling a large challenge in of itself. The simulation results have been used to generate synthetic catalogs for large-scale structure surveys, including DESI and eBOSS, as well as CMB experiments. A detailed catalog for the LSST DESC data challenges has been created as well. We publicly release some of the Outer Rim halo catalogs, downsampled particle information, and lightcone data.
\end{abstract}

Subject headings: methods: N-body — cosmology: large-scale structure of the universe

\section{INTRODUCTION}

Future large-area cosmological surveys, to be carried out with, e.g., the Large Synoptic Survey Telescope (LSST) Abell et al. 2009, Abate et al. 2012), the Dark Energy Spectroscopic Instrument (DESI) (Aghamousa et al.2016), or the Wide Field

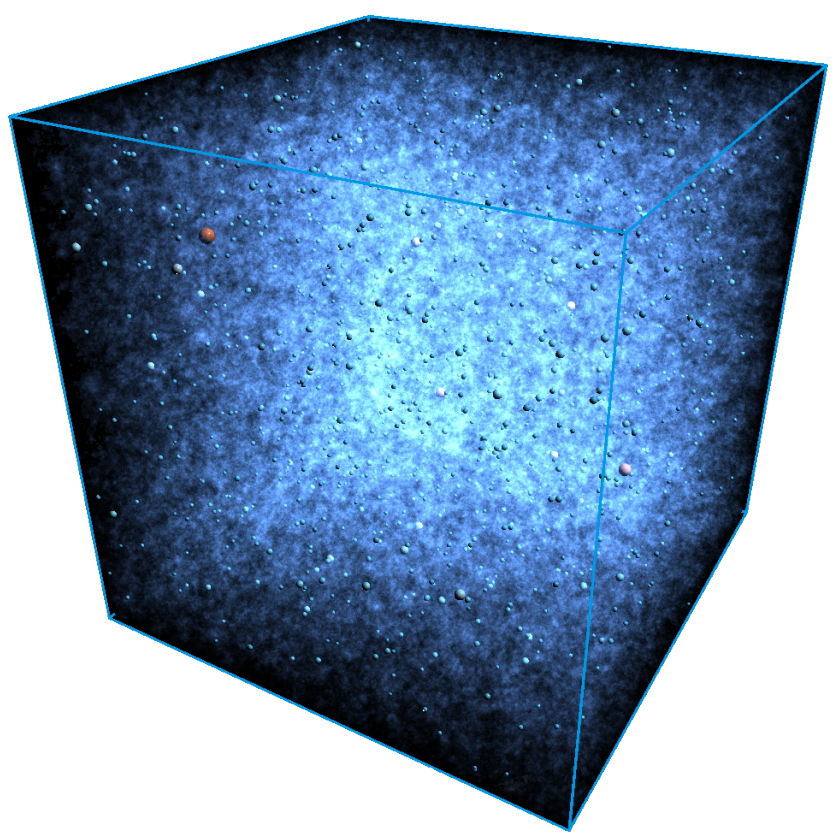

FIG. 1.- Visualization of the halos in the Outer Rim simulation at redshift $z=0$. Halos above a mass of $1.8 \cdot 10^{15} \mathrm{M}_{\odot}$ are shown as spheres, capturing $\sim 1000$ of the heaviest halos, and colored by mass (red indicates more massive), while halos down to a mass of $\sim 5 \cdot 10^{10} \mathrm{M}_{\odot}$ are represented by blue Gaussian density "splats".
Infrared Survey Telescope (WFIRST) (Gehrels 2010; Spergel et al. 2015), are becoming ever more complex as telescopes reach deeper into space, mapping out the distributions of galaxies at farther and farther distances. These observations provide a treasure trove of information about the make-up of the Universe and its evolution from the very first moments to today. Interpreting the observations and extracting knowledge from the surveys requires sophisticated simulations that track the detailed formation of structure over time.

These cosmological simulations need to cover large volumes and, at the same time, provide enough mass resolution to resolve small halos that host dim galaxies at early epochs. They enable a range of important tasks: investigations of systematic errors and possible mitigation strategies, developing and testing complex analysis pipelines and workflows, providing crucial information for survey optimization, exploring new probes and cross-correlations among different wavebands, or forecasts for future survey ideas. In addition, the simulations play important roles in furthering our understanding of the basic physics of structure formation.

In order to provide a simulation that can satisfy a substantial range of these needs, we ran the Outer Rim simulation, one of the largest N-body gravity-only simulations at the achieved resolution ever carried out. The Outer Rim simulation covers a volume of $(4.225 \mathrm{Gpc})^{3}$ and evolves $10,240^{3}$ particles, leading to a mass resolution of $\sim 2.6 \cdot 10^{9} \mathrm{M}_{\odot}$. We saved and analyzed almost 100 time snapshots, yielding a data volume of more than 5PB. The data products from the simulation include halo catalogs for different mass definitions, subhalo catalogs, detailed merger trees, two-point statistics, lightcone representations of the data, and subsamples of raw and halo particles. The high mass and temporal resolution of the simulation and the corresponding outputs make this data set ideal for survey related investigations as well as large-scale structure studies. Synthetic catalogs can be created using a variety of methods, 
from Halo Occupation Distribution (HOD) approaches to more detailed semi-analytic models. The Outer Rim run continues in the tradition of the Millennium simulation by Springel et al. (2005), with a similar mass resolution but with a volume coverage increase by more than a factor of 200. This is essential for capturing galaxy clustering at large length scales and for achieving the needed statistics for cluster cosmology.

The simulation was carried out with the Hardware/Hybrid Accelerated Cosmology Code (HACC) described in great detail in Habib et al. (2016). The Outer Rim simulation used a version of HACC that has been specifically optimized for high-performance on the BlueGene/Q (BG/Q) system Mira, using a tree implementation for the short-range solver as well as individual particle time stepping once the clustered regime is reached. Due to the vast size of the simulation, some of the analysis tasks also posed new challenges as described in this paper. Figure 1 shows an example visualization of the halos extracted from the simulation.

In this paper we describe the Outer Rim simulation and related data products in detail, some of which will be publicly released. We also discuss some of the computational implementations on the BG/Q that enabled us to carry out the simulation efficiently and how these developments also apply to nextgeneration supercomputers. Next we show a set of selected results obtained from the simulation so far. Some of these results have been used already by eBOSS (Zarrouk et al. 2018, GilMarín et al. 2018; Hou et al. 2018) and in a detailed study of the halo concentration-mass relation by Child et al. (2018); others will be discussed in more detail in accompanying papers in the near future.

The paper is organized as follows. First, in Section 2, we give a brief description of HACC, focusing on the BG/Q and therefore on the many-core optimizations employed. We also provide a short summary of how we combat the analysis workflow challenges and the I/O performance achieved on Mira. In Section 3 we describe the data products from the simulation and highlight some of results obtained so far. More detailed analyses of the data covering a wide range of scientific questions are in preparation; this first paper will serve partially as a motivating reference for these upcoming studies. In Section 4 we briefly describe the data we release from the Outer Rim. The data release is part of a larger project described in an accompanying paper. We conclude and provide a brief outlook in Section 5 .

\section{HACC}

The HACC code has been developed over the last several years (Habib et al. 2009, 2012, 2013, 2016) with the specific aim of providing a code that runs at high performance on a variety of architectures without having to implement intrusive hardware-dependent changes. As often used in cosmological N-body codes, the total force evaluation in HACC is divided into an FFT-based long-range force solver and a shortrange force solver. In the current version of HACC, most recently described in Habib et al. (2016), 95\% of the code base (the long-range force solver and the MPI-based communication layer) remains unchanged when moving between different platforms while the remaining 5\% (the on-node implementation of the short-range solver) is optimized given the specification of the hardware. This optimization includes algorithmic changes as well - depending on costs of computation vs. data movement, in some cases tree-based algorithms are optimal, while in other cases direct particle-particle interactions may be prefer- able. Given that most of the code stays unchanged, we refer to this approach as "soft-portability", compared to a hard-portable code that stays completely unchanged and the compilers on the different systems take care of all the optimization. Currently, HACC itself, in this sense, is soft-portable, while the analysis set up is hard-portable, relying on the NVIDIA Thrust library.

Originally developed for the Cell-accelerated machine Roadrunner (Habib et al. 2009, Pope et al. 2010) - keeping softportability in mind even at that early stage - HACC currently runs on a diverse range of architectures, including $\mathrm{X} 86, \mathrm{BG} / \mathrm{Q}$, GPU-accelerated systems, and most recently Intel's Knights Landing (KNL), which powers the most recent supercomputers installed at ALCF and NERSC. Habib et al. (2012) provide some details about the original BG/Q implementation and optimization while Habib et al. (2013) focus on the portability aspect of HACC and demonstrate outstanding performance on Titan, a GPU accelerated supercomputer. In the following we focus on the BG/Q specific aspects of the HACC short-range solver, given that the Outer Rim simulation was carried out on Mira, the BG/Q system at the ALCF. For a detailed discussion of the implementation of the long-range solver, the reader is referred to Habib et al. (2013). We also comment on how our effort on the BG/Q translates into a rather straightforward port to KNL-based systems.

\subsection{HACC on Many-Core Systems}

In this section, we focus on the on-node short-range solver for many-core systems implemented in HACC. The short-range solver in this case uses a tree-based algorithm. In order to further increase parallelism, and to decrease the amount of pointer-chasing relative to numerical computation during the short-range force calculation, we implemented a scheme which builds multiple recursive-coordinate-bisection (RCB) trees (or "bushes") on spatially-disjoint regions instead of one large tree covering the entire volume of one rank. A physical scale is chosen, between $1-2 h^{-1} \mathrm{Mpc}$ for the Outer Rim simulation, depending on the clustering state, and the particles are first sorted into bins associated with chaining mesh (CM) cells on a regular spatial grid. Each cell, boundary cells excluded, are cubic

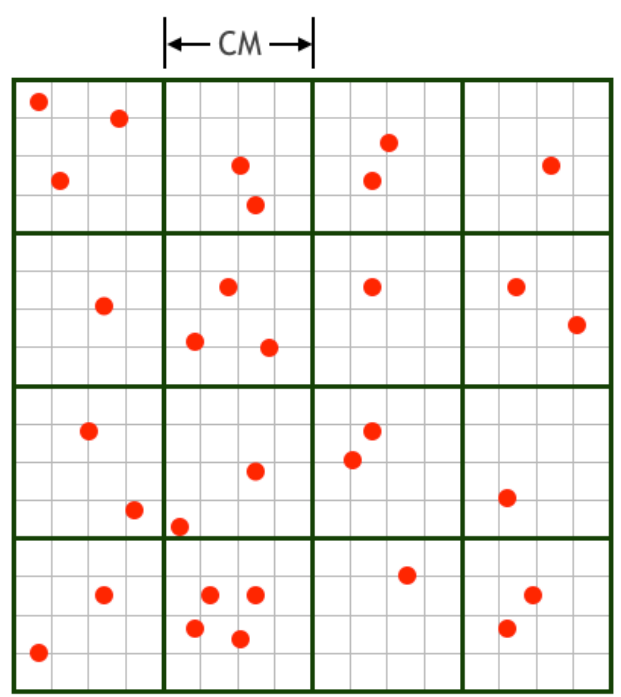

FIG. 2.- Simplified schematic view of a single-rank tree structure shown in two dimensions. HACC has two parameters that can be tuned to optimize time-to-solution on many-core systems, the chaining mesh (CM) size and the final number of particles on a leaf at which the tree bisection stops. 
with a linear size of the chosen scale. Within each cell, an RCB tree is built. Figure 2 shows a simplified schematic view of this scenario in two dimensions. The chaining mesh cell size and the number of particles per leaf node at which to stop the tree bisection are input parameters to the code. These two parameters provide significant flexibility in tuning the short-range solver on different architectures for achieving the fastest timeto-solution. In general, higher clustering at late times is handled better with bigger chaining mesh sizes, while less clustered distributions require a smaller chaining mesh size. We accordingly adjust the CM scale during the course of the simulation. The chaining mesh building process is trivially parallelizable because the cells are spatially disjoint, and this parallelization is important to the overall thread scaling of the code. The individual trees are also less deep, especially those without many particles in the clustered regime, which might be trivial, providing a small practical performance advantage. This is true even though, for each leaf node in each RCB tree, forces must be accumulated from 27 trees (the parent tree itself plus 26 neighbors). Each tuple of <leaf node, neighboring tree $>$ is added to a work queue, and this queue is processed by all threads using a dynamically-scheduled OpenMP parallel loop. Because multiple threads might end up trying to update forces on particles in the same leaf node concurrently, locks are used to protect the updates. For the Outer Rim simulation, one lock per tree was used.

The transition from the BG/Q architecture to the KNL systems was straightforward. One lock per leaf node was used to decrease lock contention and increase thread scalability. Prior to work on KNL systems, a version of the pair-wise short-range particle interaction kernel had been implemented for the AVX2 instruction set using intrinsics, relying heavily on the experience gained from developing the QPX intrinsics kernel for the BG/Q architecture. For KNL systems, the AVX2 intrinsics were replaced with the corresponding AVX512 intrinsics, with some minor changes to take advantage of an improved inverse square-root instruction available on KNL, and the optimal inner loop unrolling factor was determined experimentally. Finally, a number of tests were run to optimize the balance of MPI ranks and OpenMP threads per node and to tune the parameters that determine the spatial extent and depth of the RCB trees.

\subsection{I/O Performance on the Mira GPFS File System}

In order to obtain optimal $\mathrm{I} / \mathrm{O}$ performance on a range of large-scale parallel file systems, we developed a custom I/O implementation for HACC, called GenericIO. GenericIO is a write-optimized library for writing self-describing scientific data files and is publicly available $\sqrt{1}$ Mira is connected to an IBM General Parallel File System (GPFS). Optimal I/O timing for the raw particles was obtained when writing out the data into 256 files. The write-speed was approximately $0.15 \mathrm{~TB} / \mathrm{s}$ which allowed us to write raw particle files in just under 5 minutes. In our current implementation, the number of analysis files written during in-situ analysis is locked to the same number of files as the raw outputs. At early steps this can lead to a relatively large number of small files, e.g., the number of halos is tiny at very high redshifts and most files therefore will be empty. This approach then leads to rather slow $\mathrm{I} / \mathrm{O}$ and we switch from Posix-I/O to collective MPI-IO by providing a switch coupled to the size of the output. For outputs smaller than a certain size, MPI-I/O is used while for large files Posix-I/O is chosen. The

\footnotetext{
${ }^{1}$ http://trac.alcf.anl.gov/projects/genericio
}

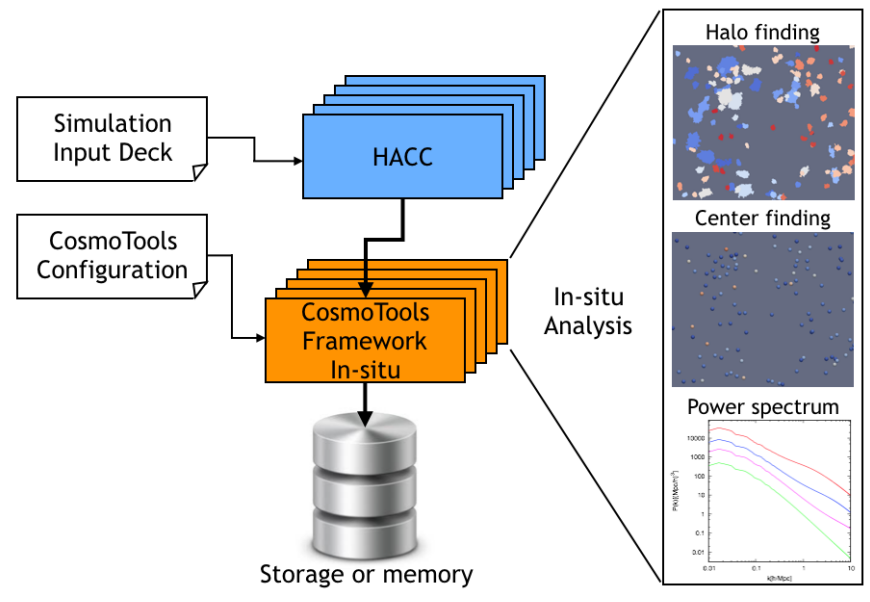

FIG. 3.- Schematic description of our current workflow implementation in HACC if the analysis is run in-situ. In addition, raw HACC data can be written directly to storage and CosmoTools can be run on it later. When carrying out the Outer Rim simulation, CosmoTools was in an early stage of development and has been considerably enhanced since then.

file size above which to use Posix-I/O is an easily adjustable parameter in the HACC input deck. A more detailed description of GenericIO including comparison to the performance of PNetCDF is given in Habib et al. (2016).

\subsection{Analysis Workflow}

A simulation of the size described in this paper poses an enormous challenge with regard to analysis tasks, in particular where the raw particle data is concerned. Due to memory demands, handling the raw data usually requires as much of the resource as running the simulation itself (in the specific case described in this paper, 32 racks of Mira, out of a total of 48) and therefore is extremely expensive. Just reading in the data for analysis even with a highly optimized I/O approach that we developed for Mira, costs close to 200k core hours for a run of the size of the Outer Rim simulation, equivalent to running a medium-size simulation. Therefore, if possible, it is important to avoid reading in the raw data and instead to carry out analysis tasks on the fly while the raw data is still available in memory. This in turn requires writing fast, load-balanced, and memory efficient analysis tools. As part of HACC we have developed and continue to enhance CosmoTools, a library of highperformance analysis tools that can be run in-situ or off-line (for a description of an earlier version, see Habib et al. 2016). As shown in Figure 3, CosmoTools is triggered from the simulation input deck by a simple on/off switch and the specification of the analysis input parameter file, the CosmoTools configuration file. This configuration file contains a list of the tools to be run, the list of snapshots when they are run, and the tool specific parameters, such as linking length, minimal halos mass, or mass definition for the halo finder. It is also possible to exclude analysis tools when compiling and building HACC. This option is included to allow the use of external libraries within analysis tools. The advantage of external libraries is that they are often highly tuned to deliver good performance, the disadvantage is that they might not be available on the platform the simulation is run on. In order to guard for this possible problem, analysis tools can be excluded during the build process. (An example for this is a density estimator that depends on the Qhull library.)

Throughout the simulation, we periodically (in-situ) evaluate the matter power spectrum; this calculation is rather inexpen- 
sive but very helpful to monitor the health of a run. The halo finder is also frequently run in-situ. As we describe in the next section, halo finding is carried out including a range of subanalysis tasks beyond the halo finding such as detailed tracking of halo cores over time and saving all particle identifiers ("IDs") of particles that reside at some point in halos. Storing all this information allows us to build many more data products offline without requiring very large resources since the halo finding step reduces the data amount considerably. It also ensures enough flexibility to carry out analyses on the data products exploring different parameter settings. This opportunity is very important since with a simulation like the Outer Rim run, we entered new regimes for a number of analysis tasks.

In the case of high mass resolution simulations at late times, when large clusters have formed, some of the halo analysis tasks, like evaluating the halo gravitational potential minimum for center finding, can become rather expensive. As detailed in Sewell et al. (2015) we therefore implemented a workflow option where only the small and medium size halos are analyzed in-situ while all halo particles from the very large halos are written out to disk and are then analyzed in post-processing. Usually, only a very small percentage of the halos will be analyzed off-line this way (so storage requirements are minimal) but the approach saves a substantial amount of computer time. Sewell et al. (2015) provide detailed timing information based on a large simulation and show different analysis workflows to optimize the the usage of core hours.

For the Outer Rim simulation we started the halo finder insitu but encountered some memory bottlenecks when reaching lower redshifts, demonstrating that an extreme simulation like this one always uncovers new challenges that were not apparent when carrying out medium-scale simulations. Once the stage was reached where cluster sized halos are forming we switched to off-line analysis in order to further develop the approach described above where we divide halos into two mass classes and analyze them separately. In the meantime we also improved the memory efficiency of the halo finder considerably to enable the analysis of the final time snapshots.

\section{THE OUTER RIM SIMULATION}

In this section we provide details about the simulation, including a list of saved outputs and common statistics measured at various redshifts. The simulation used 32 racks of Mira, the BG/Q supercomputer at the Argonne Leadership Computing Facility, each rack hosting 1024 nodes, each node consisting of 16 user-accessible cores. During the earlier phase of the simulation, we used 8 ranks per node and 8 threads, while we switched to 1 rank per node and 64 threads during the later, highly clustered stages of the simulation, approximately at $z \sim 0.6$. At this point we also switched on the individual particle time stepper instead of evolving each particle with the same number of time steps.

The overall size of the dataset generated exceeds 5PB, providing an unprecedented amount of detailed information about the formation of the structures in the simulation over time. We classify our outputs into three levels. Level 1 data is the raw particle data or density and potential fields that cover the full volume as well as summary statistics obtained directly from the raw data, such as the matter power spectrum. Level 2 data is obtained by carrying out an analysis step on Level 1 data to generate, e.g., halo catalogs and summary statistics describing the Level 2 data, such as mass functions. Level 2 data is usually at least an order of magnitude smaller than Level 1 data.
Finally, Level 3 data is derived from Level 2 data and often already closer to actual observables, such as galaxy catalogs or sky maps at different wavelengths. In the following we will specify what level the data products belong to explicitly.

\subsection{Parameters}

The cosmology used for the simulation is close to the bestfit model determined by WMAP-7 (Komatsu et al. 2011). This is the same cosmology underlying two related simulations, the Q Continuum simulation, described in Heitmann et al. (2015) and the MiraU simulation. The MiraU simulation was used for example in Flender et al. (2015) to investigate and model the kinematic Sunyaev-Zel'dovich effect and in Heitmann et al. (2016) to study requirements for precision predictions for nextgeneration dark energy surveys. This set of simulations covers a range of mass resolutions, from $\sim 10^{8} \mathrm{M}_{\odot}$ to $\sim 10^{10} \mathrm{M}_{\odot}$ enabling different science use cases and allowing for a number of convergence tests.

The chosen cosmological parameters are: $\omega_{\mathrm{cdm}}=0.1109$, $\omega_{\mathrm{b}}=0.02258, n_{s}=0.963, h=0.71, \sigma_{8}=0.8, w=-1.0$. The volume of the simulation is $V=(4225.35 \mathrm{Mpc})^{3}=\left(3000 h^{-1} \mathrm{Mpc}\right)^{3}$, with $10,240^{3}=1.07$ trillion particles. This results in a particle mass of

$$
m_{p}=2.6 \cdot 10^{9} \mathrm{M}_{\odot}=1.85 \cdot 10^{9} h^{-1} \mathrm{M}_{\odot} .
$$

The size of the simulation was chosen to cover a volume large enough to enable the generation of synthetic sky catalogs for surveys such as DESI and LSST and at the same time to have enough mass resolution to capture halos reliably down to small masses. The simulation was started at $z_{\text {in }}=200$ and the initial conditions were generated using the Zel'dovich approximation (Zel'dovich 1970). The transfer function was generated with $\mathrm{CAMB}$ (Lewis et al. 2000). This leads to the same general simulation set-up in all simulations mentioned above, Outer Rim, Q Continuum, and MiraU.

\subsection{Particle Outputs - Level 1 Data}

As for the Q Continuum and MiraU simulations, we store a large number of time snapshots between $z=10$ and $z=0$. Originally, we saved 101 snapshots evenly spaced in $\log _{10}(a)$, but two snapshots were unfortunately corrupted on disk - an occupational hazard - before they were fully analyzed. The final list of 99 outputs in redshift $z$ is:

$$
\begin{aligned}
& z=\{10.04,9.81,9.56,9.36,9.15,8.76,8.57,8.39,8.05,7.89, \\
& 7.74,7.45,7.31,7.04,6.91,6.67,6.56,6.34,6.13,6.03,5.84, \\
& 5.66,5.48,5.32,5.24,5.09,4.95,4.74,4.61,4.49,4.37,4.26, \\
& 4.10,4.00,3.86,3.76,3.63 .3 .55,3.43,3.31,3.21,3.10,3.04, \\
& 2.94,2.85,2.74,2.65,2.58,2.48,2.41,2.32,2.25,2.17,2.09, \\
& 2.02,1.95,1.88,1.80,1.74,1.68,1.61,1.54,1.49,1.43,1.38, \\
& 1.32,1.26,1.21,1.15,1.11,1.06,1.01,0.96,0.86,0.82, \\
& 0.78,0.74,0.69,0.66,0.62,0.58,0.54,0.50,0.47,0.40, \\
& 0.36,0.33,0.30,0.27,0.24,0.21,0.18,0.15,0.13,0.10,0.07, \\
&0.05,0.02,0.00\} .
\end{aligned}
$$

For each particle in these snapshots we store position, velocity, and a unique particle ID to enable tracking particles over time. Each snapshot encompasses roughly 40TB of data.

Due to the wide variety of science projects enabled by a large simulation, storing as much of the raw particle outputs as possible is desirable. However, due to the large amount of data 


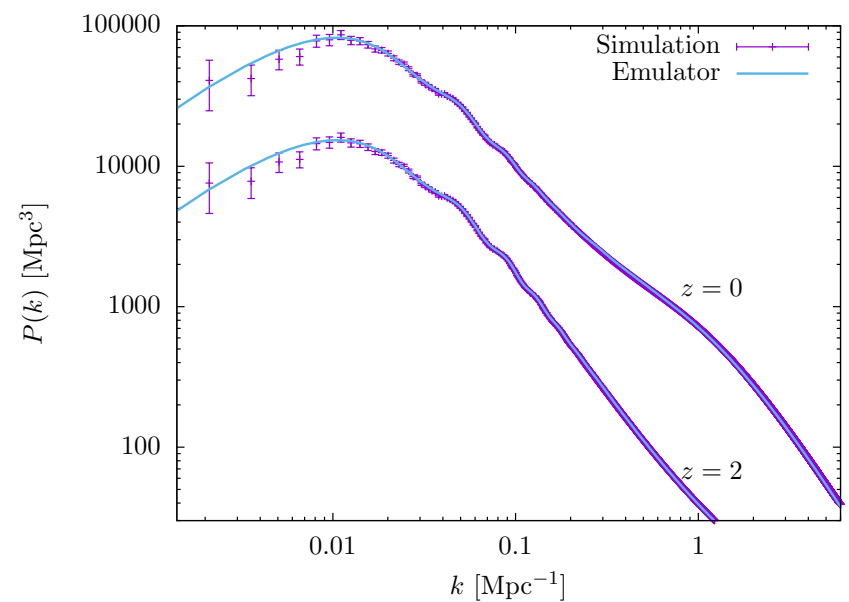

FIG. 4.- Power spectrum results at redshift $z=0$ and $z=2$. For comparison, we show predictions from the Cosmic Emulator by Heitmann et al. (2014).

that is produced it is not practical to keep the data for a long period of time on spinning disk. In addition, data can get corrupted on disk. Therefore, archiving data on long-term storage is important: For the Outer Rim simulation, we use ALCF's and NERSC's tape storage system, which employs the HighPerformance Storage System (HPSS). Both facilities have enabled Globus ${ }^{2}$ on both disk and tape storage system to allow easy transfer of the data. During the period of running and analyzing the simulation, we lost two snapshots on disk due to irreparable disk failures and also had several corrupted failures on tape. A second copy on an independent tape system therefore appears to be very important if one wants to guarantee the availability of the full data set over an extended period of time.

In addition to the full particle data sets, we also save for each time snapshot a randomly selected set of particles that comprises $1 \%$ of a full snapshot. The down-sampled data sets are sufficient to measure correlation functions and generate lightcone density maps for weak lensing ray-tracing applications. The down-sampled data are kept on disk for easy and fast access for post-processing analysis.

\subsubsection{Matter Power Spectrum}

The matter power spectrum was generated in-situ at many time snapshots during the simulation. HACC is set up to automatically produce a power spectrum before the end of a submission cycle and before every check-point restart data dump. This allows for easy inspection of the state of the simulation and whether the evolution is progressing as expected. In addition, for each time step at which we save a particle snapshot we evaluate the matter power spectrum. Currently, the in-situ power spectrum calculation is using the same grid size as the longrange PM solver used in the simulation (in the current case, this is a $10,240^{3}$ grid). Each power spectrum evaluation took approximately a minute. Figure 4 shows the power spectrum for redshifts $z=2$ and $z=0$ from the Outer Rim simulation. The large volume of the simulation enables an excellent resolution for the baryonic oscillation region (the "wiggly" region). We also show the predictions from the Extended Cosmic Emulator, published in Heitmann et al. (2014). As for our other simulations, Q Continuum and Mira-Titan Universe, the agreement is very good and at the expected level of accuracy following Heit-

${ }^{2}$ https://www.globus.org/ mann et al. (2014).

\subsection{Friends-of-Friends Halo Outputs - Level 2 Data}

Halo catalogs using different halo mass definitions were generated and stored at each time snapshot in order to have sufficient information to build detailed merger trees during the post-processing phase using a new merger tree construction code (Rangel et al. 2017). In the following we provide details about the relevant data products.

\subsubsection{Friends-of-Friends Catalogs and Halo Particle Information}

The friends-of-friends (FOF) halo catalogs were generated using a linking length of $b=0.168$. We store a large number of halo properties - halo position and velocity based on the halo minimum potential as well as the center-of-mass, the halo mass, angular momentum, and kinetic energy for all halos with at least 20 particles. The FOF finder follows the standard implementation of identifying all particles that reside within a certain distance (the linking length) of a particle and then the neighbors of those particles, etc. The linking length is defined with respect to the mean inter-particle spacing. This group finding algorithm was first used in cosmology by Davis et al. (1985). The FOF algorithm is fast and makes no assumptions about the halo geometry; it is also completely reproducible - two FOF finders run with the same linking length should give exactly the same result. Our FOF halo finder implementation (Woodring et al. 2011 ) is based on a very fast, tree-based serial halo finder that is then parallelized by overloading each rank with a sufficiently large border from the neighboring ranks to ensure that halos that extend beyond one rank are successfully found. After all halos are found on all ranks, halos that have been found more than once (due to the overloading) are eliminated. In addition to the "overloaded" distribution of the particles, this is the only step in the halo finding that requires communication between ranks.

The centers of the halos are determined by the location of the FOF halo's minimum gravitational potential, where the potential at a given halo particle is obtained by finding the distance $r$ to every other halo particle and then accumulating all of the values of the negative of mass divided by the distances to the particle. The center-of-mass and the halo velocities are obtained by simply summing over all positions and velocities and dividing by the number of particles. The FOF halo mass is determined by the number count of particles in each halo. Additional properties we store for each halo are the angular momentum, $\mathbf{L}=\sum_{i} m_{i}\left(\mathbf{r}_{i} \times \mathbf{v}_{i}\right)$ and the kinetic energy, $E=0.5 \sum_{i} m_{i} \mathbf{v}_{i}^{2}$. The implementation of the halo finding algorithm and center finder are described in detail (including timings for the very high mass resolution Q Continuum simulation) in Sewell et al. (2015). We follow a similar strategy for the halo center finding as outlined in Sewell et al. (2015) for the last 14 time steps. We use 32 racks of Mira to find all the halos and determine halo centers for halos up to 100,000 particles. For the more massive halos, we write out the complete list of halo particles and run the center finder on those outputs on only 8 racks. This reduces the computational time considerably.

In addition to the halo catalogs themselves we also store all the unique particle IDs and the associated halo id, if the particle belongs to a halo. This information allows us to build halo merger trees in post-processing explicitly following each particle's complete history. We also save all the particles that reside 


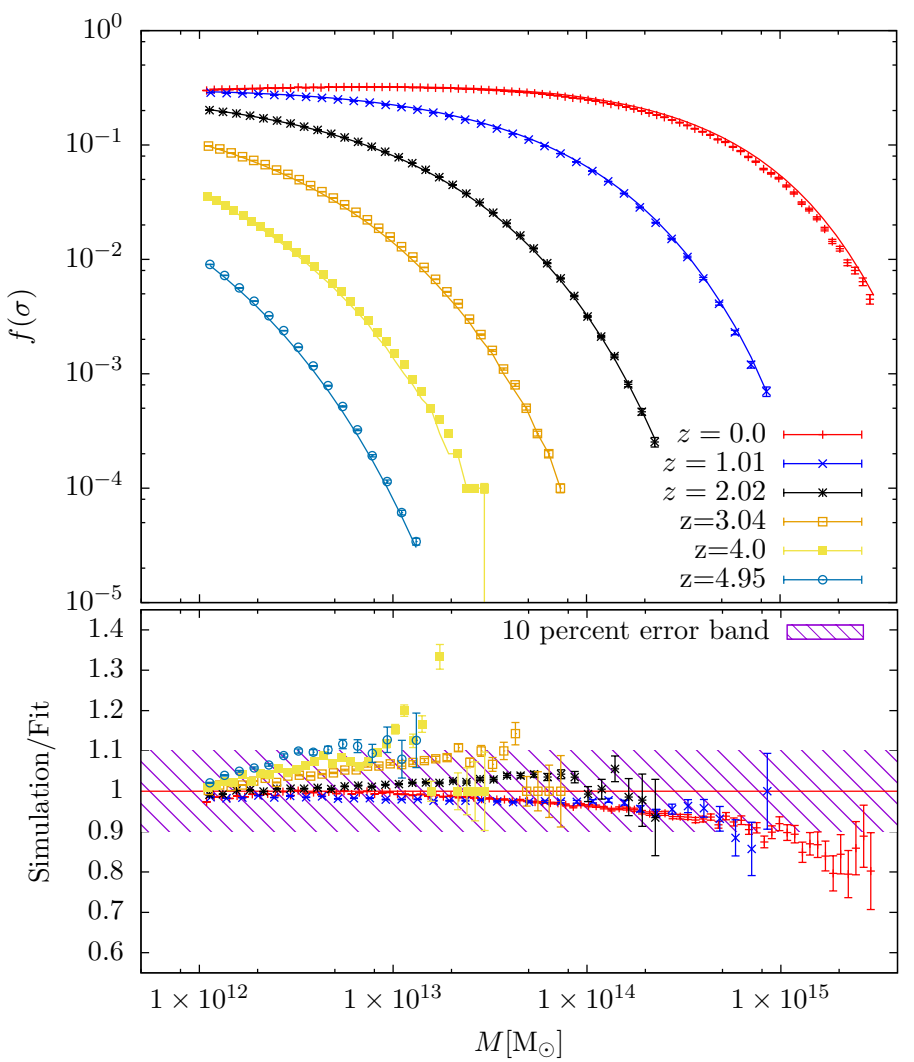

FIG. 5.- Upper image: Halo mass function $f(\sigma)$ as a function of halo mass at five redshifts. We show the measurements from the simulations (including statistical error bars) as well as the fit derived by Bhattacharya et al. (2011). The mass definition used here is FOF with $b=0.2$. The lower image shows the ratio of the simulation result and the fit. We also mark a $10 \%$ band that covers most of the results.

in halos with at least 100,000 particles (including position and velocity information for all particles) and a random selection of $1 \%$ of all halo particles, but at least 5 particles per halo. This information is useful when implementing HOD or similar galaxy models, allowing the placement of model galaxies onto particles directly, if so desired.

Finally, we also store a handful of catalogs built with a linking length of $b=0.2$. In particular, we generated catalogs at $z=\{4.95,4.0,3.04,2.02,1.006,0.0\}$ with a minimum number of 20 particles per halo. The main reason for analyzing halos with this specific linking length is the existence of many results in the literature for $b=0.2$, in order to compare our results with earlier investigations. It also allows us to check the universality of the mass function that was obtained in earlier work for this linking length over a wide variety of redshifts. More details about the mass function are given in Section 3.3.2.

\subsubsection{Friends-of-Friends Halo Mass Function}

Next we show results for the halo mass function as measured by an FOF halo finder with a linking length of $b=0.2$ to compare with a number of previous results obtained using this definition and because of the near-universality of the mass function obtained using this definition, as first discussed in depth by Jenkins et al. (2001). Figure 5 shows the differential mass function $f(\sigma, z)$ as introduced by Jenkins et al. (2001) as a function of halo mass $M$ for redshifts between $z=4.95$ and $z=0$ :

$$
f(\sigma, z)=\frac{d \rho / \rho_{b}}{d \ln \sigma^{-1}}=\frac{M}{\rho_{b}(z)} \frac{d n(M, z)}{d \ln \left[\sigma^{-1}(M, z)\right]} .
$$

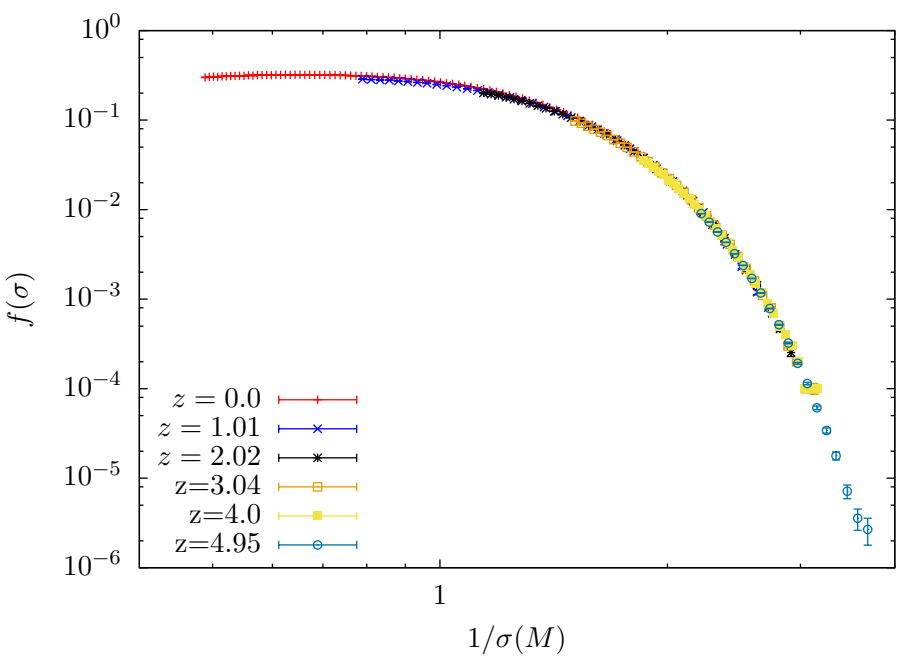

FIG. 6.- Universality of the mass function across redshifts out to $z \sim 5$. We follow the behavior of the mass function $f(\sigma)$ as a function of $1 / \sigma(M)$ across a wide range of redshifts to demonstrate the remarkable power of universality.

Here, $n(M, z)$ denotes the number density of halos with mass $M$, $\rho_{b}(z)$ is the background density at redshift $z$, and $\sigma(M, z)$ is the variance of the linear density field.

The agreement across a wide range of redshifts over a broad dynamic range (with the very small statistical errors) testifies to the power of the universal description.

As is now commonly done for FOF halos (see, e.g., Bhattacharya et al. (2011) for detailed tests) we include a mass correction for low mass halos to account for finite sampling bias of the form:

$$
n_{h}^{\text {corr }}=n_{h}\left(1-n_{h}^{-0.65}\right) .
$$

We only include bins with at least 100 halos. Besides the simulation results, we also present a mass function fit derived in Bhattacharya et al. (2011), including a simplification for the redshift dependence suggested in Heitmann et al. (2015). The fit is given by:

$$
f(\sigma, z)=A \sqrt{\frac{2}{\pi}} \exp \left[-\frac{a \delta_{c}^{2}}{2 \sigma^{2}}\right]\left[1+\left(\frac{\sigma^{2}}{a \delta_{c}^{2}}\right)^{p}\right]\left(\frac{\delta_{c} \sqrt{a}}{\sigma}\right)^{q},
$$

with the parameters:

$$
A=\frac{0.333}{(1+z)^{0.11}} ; \quad a=0.788 ; \quad p=0.807, \quad q=1.795 .
$$

The density threshold for spherical collapse, $\delta_{c}=1.686$, is taken to be the same for all redshifts.

The symbols in the upper panel in Fig. 5 show the measurements from the simulation, including statistical error bars, while the lines show the mass function fit as given in Eqs. (5. 6). The lower panel in Fig. 5 shows the ratio of the simulation results and the mass function fit. The shaded region marks a deviation of $10 \%$ between fit and simulation. In the cluster mass regime at $z=0$, the measured mass function deviates from the fit at the $10 \%$ level. This deviation is consistent with earlier findings by, e.g., Crocce et al. (2010), who carefully compared different box size simulations and find differences in the mass function for a $3^{-1} \mathrm{Gpc}$ box compared to $4.5^{-1} \mathrm{Gpc}$ and $7.68^{-1} \mathrm{Gpc}$ boxes at a similar level as we find here. In order to demonstrate the almost universal form of the mass function more concretely, we also plot $f(\sigma, z)$ as a function of $1 / \sigma(M)$ in Fig. 6 for all six redshifts between $z=4.95$ and $z=0$. 


\subsubsection{Halo Core Catalogs}

The halo formation history plays a crucial role in influencing the properties of the galaxies hosted by a particular halo (see, e.g., Wechsler \& Tinker (2018) for a recent review). In particular, the infall mass of a halo when joining another halo and becoming a part of it, either in the form of a subhalo or simply in the form of additional, disrupted dark matter mass, is important to follow over time. In order to fully keep track of this information, including the afterlife of a halo once it has fallen into another halo, we introduce the notion of a halo "core". For each halo that is larger than 100 particles at a time step $i$, we determine the position and velocity of the ten particles closest to the center, the halo core particles. We store a halo core file for each time snapshot reporting all halo core particles, including unique particle IDs. In addition, at each of the remaining time steps, we identify the positions of all the core particles from all the previous time snapshots and store them in an accumulated core particle file. If a particle is still a core particle in step $i+j$ (for example the halo at step $i$ never merged or fell into another halo but rather just accreted mass) we only record the core particles once in the accumulated core particle file. The accumulated core particle files allow us to track halos over time in great detail. The advantage of the core tracking over simple subhalo merger trees is that we retain the information of disrupted halos as well. We carried out detailed tests on a smaller simulation with similar mass resolution to confirm that ten core particles provide this information reliably. We will present a detailed study of the core particle tracking and comparison to subhalo identification in a forthcoming paper.

Figure 7 shows an example of the cores identified in a cluster sized halo of mass $\sim 10^{15} \mathrm{M}_{\odot}$. Each point shows a core that originated at some point from a 100 particle halo. The cores are colored with respect to their infall time while the size signals the radius of the core (magnified by a factor of 100 for visibility). The cores close to the center belong the longest to the mother halo as one would expect. Most halos on the outskirts joined the main halo at later times. Figure 8 shows the trajecto-

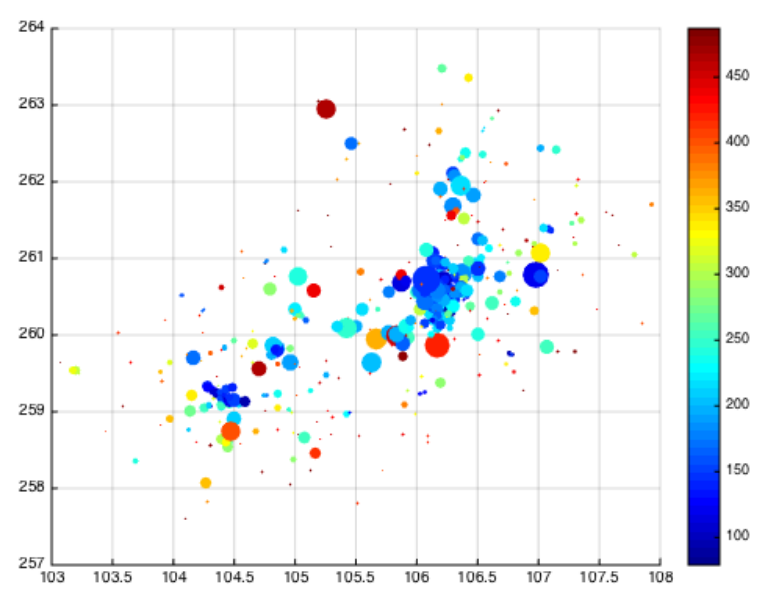

FIG. 7. - Halo cores found in a cluster sized halo with a mass of approximately $10^{15} \mathrm{M}_{\odot}$ at redhift $z=0$. The core of each halo with more than 100 particles that fell into this cluster at some point in time is marked with a point. The point size is 100 times the core radius and the color indicates the redshift of infall. As expected from hierarchical structure formation, the center of the cluster is populated with cores from very early times while the outskirts are mostly populated with halo cores that more recently fell into the cluster.

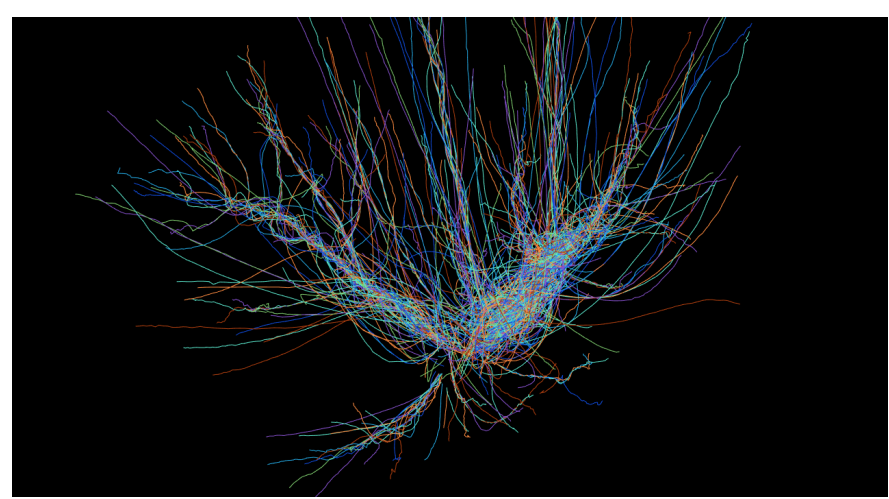

FIG. 8. - Halo core trajectories followed over time leading to one massive halo at $z=0$. Each line tracks the evolution of a core from a halo that had at least 100 particles at formation time until its infall into the main halo.

ries of cores over time joining to merge in one large halo at the end. Each core trajectory follows a halo of at least 100 particles until it falls into another halo.

\subsubsection{Subhalo Catalogs}

In addition to the halo catalogs, we have also identified subhalos for cluster sized halos with more than 100,000 particles for redshifts between $1.5 \leq z \leq 0$. The redshift range and mass cut are chosen so as to model clusters as observed by the South Pole Telescope (SPT) and described in the SPT cluster paper by Bleem et al. (2015). We find subhalos by combining a local density estimator with phase space information. After building a list of possible subhalo candidates we determine if a particle is gravitationally bound to the subhalo; if it does not belong to the subhalo, it is assigned to the main halo. We then keep only subhalos that have at least 20 particles. We have used this approach in $\mathrm{Li}$ et al. (2016) to find subhalos and then populated three clusters from the Outer Rim simulation with cluster galaxies. We generated realistic strong lensing images from these clusters emulating the known properties of different telescopes. With the complete set of clusters, including subhalo information, we will extend the work described in Li et al. (2016) in the near future to a complete SPT cluster sample. In addition, the subhalo information will be used for comparison with the core tracking approach described in the previous section. Preliminary results show that intact cores within major halos can be mapped onto subhalos found by the subhalo finder. A detailed analysis of these results is in preparation.

\subsubsection{Merger Trees}

In addition to the halo core tracking, we also built traditional merger trees on the FOF halo catalogs. We take into account each halo with at least 20 particles and follow its evolution over time. For each halo at $z=0$ we have a complete merger history as far back into the past as $z=10$. As is well known, building halo merger trees on a number of discrete time snapshots is very challenging. Halos fall in and out of existence due to an unavoidable lower mass bound, there are halo merging and splitting events, and fly-by events where parts of halos may get disrupted. It is therefore important to build merger trees taking into account not only two neighboring time snapshots but by following the evolution of the halos over several time steps. In addition, the enormous amount of data that has to be processed for a simulation like the Outer Rim simulation requires careful 
implementation of the merger tree algorithm including an optimized load-balancing scheme. Our methodology, and initial results, are described in Rangel et al. (2017); a longer paper is in preparation.

\subsection{Overdensity Catalogs - Level 2 Data}

In addition to the FOF catalogs, we also store halo catalogs with overdensity masses. For each of the 99 snapshots we measure overdensity masses $\mathbf{M}_{200 c}$, defined with respect to 200 times the critical density. The halo location is based on FOF halo centers (determined from the potential minimum center, as described above) and SO masses are determined for halos with at least 1,000 particles. (The SO halo itself is measured from the full particle output, not just from the FOF particles.) We store a range of halo properties including the radius, the mass, the kinetic energy and angular momentum, as described previously (but now calculated from the SO halo particles), the halo concentration, the halo profile, and the velocity dispersion. For all snapshots out to $z \sim 1.5$ we also measure $\mathrm{M}_{500 c}$, again with a view of generating a cluster catalog to match the SPT survey.

\subsubsection{Concentration-Mass Relation}

We have measured the concentration of all $\mathrm{M}_{200 c}$ halos with more than 1000 particles; different concentration measurement approaches and results over a wide range of redshifts are discussed in Child et al. (2018), where we also carry out a detailed analysis of possible systematics due to the set up of the simulation (e.g., starting redshift, time stepping, halo centering) and use results from a range of simulations, including the Q Continuum and the MiraU simulations. Child et al.(2018) provides comparison with observational results as well as with fitting functions from a range of previous simulation based studies.

\subsection{Lightcone Catalogs and Maps - Level 3 Data}

The large range of Level 1 and Level 2 data allows us to generate synthetic sky maps at different wavelengths. Figure 9 shows the visualization of a subset of the lightcone data from our $\mathrm{M}_{500 c}$ catalog. We show the data out to a moderate redshift of $z \sim 0.13$ (close to what the Sloan Digital Sky Survey main sample covered) and reduce the number of halos down to 6 percent of the data from the full sphere to visually emphasize the structure of the cosmic web. The full dataset is currently used for a major strong lensing project including data from the South Pole Telescope and follow-up optical observations. For a recent paper on generating strong lensing images from the Outer Rim simulation, see Li et al. (2016), and for the investigation of the influence of line of sight halos on the detectability of clusterscale strong lensing, see Li et al. (2018). Based on this work, a large set of strong lensing images has been generated and predictions for the number of strong lenses that should be seen by SPT and follow-up optical observations are being derived and compared to the actual observations.

We have also recently generated optical catalogs using a new approach that combines empirical and semi-analytic methods. The new method makes highly efficient use of compute resources by leveraging results from smaller, high-resolution simulations into very large volume, high-resolution simulations like the Outer Rim simulation. The concept is rather straightforward, the details of the implementation will be described in a forthcoming paper. The empirical approach is used to populate the halos in the Outer Rim simulation with galaxies to

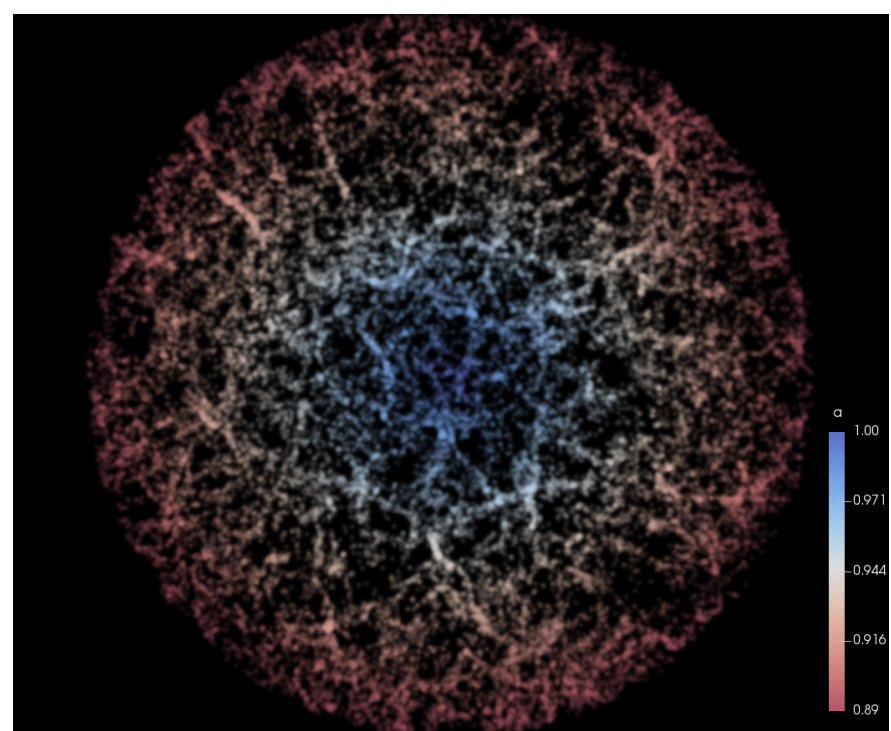

FIG. 9.- Visualization of the $\mathrm{M}_{500 c}$ lightcone catalog out to redshift $z \sim$ 0.13 and downsampled to 6 percent of the data to enhance the visibility of the cosmic web. The data is colored with respect to the value of the scale factor $a$ for each halo.

obtain the correct clustering statistics and to assign basic properties such as colors to match to observational data. Then the resulting galaxies are matched up with galaxies from a comprehensive catalog of galaxies generated with a semi-analytic approach to create a final catalog with a rich set of galaxy properties. For the semi-analytic part, we use Galacticus, developed by Benson (2012). Galacticus follows the evolution of each halo over time (in post-processing) and by applying a range of well-motivated models mimics the astrophysical processes that will lead to the formation and evolution of galaxies. We ran Galacticus on a downscaled version of the Outer Rim simulation $\left(\mathrm{a}\left(256 h^{-1} \mathrm{Mpc}\right)^{3}\right.$ volume with $1024^{3}$ particles) and generated a galaxy library this way out to $z=3$. The size of the Outer Rim simulation allows us to generate a large optical catalog employing this new approach.

The first use of the resulting optical catalog will be for the LSST Dark Energy Science Collaboration data challenges. These data challenges are full end-to-end simulations of LSST catalogs, starting from an N-body simulation, through image simulation tools, and finally include processing with the LSST data management stack. The full effort will be described in detail elsewhere. In addition, the simulation has been used to generate mock catalogs for DESI for testing fiber assignment algorithms and to optimize the DESI survey strategy (for an image of the catalog data see Habib et al. 2016). The catalog generation for this was based on a halo-occupancy distribution (HOD) approach. The eBOSS collaboration has created mock catalogs for their analysis using an HOD approach for a variety of projects. These projects include the clustering measurement of quasars by Zarrouk et al. (2018) via the correlation function and by Gil-Marín et al.(2018) via the power spectrum and by Hou et al. (2018) focusing on the anisotropic clustering of the eBOSS quasar sample in configuration space.

Beyond optical synthetic maps, the Outer Rim simulation also lends itself to generation of maps for CMB observations. Figure 10 shows an example for maps of the kinematic and thermal Sunyaev-Zel'dovich effect. The map making process is based on the procedure described in detail in Flender et al. 
OuterRim kSZ

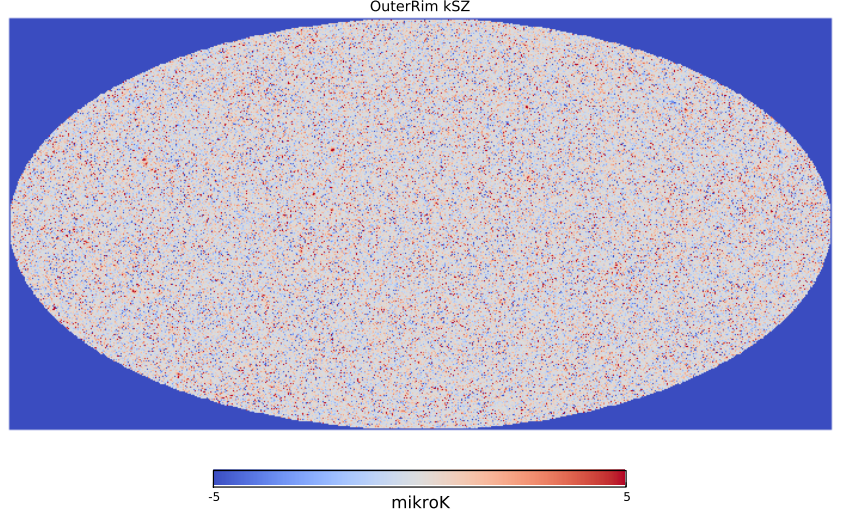

OuterRim tSz

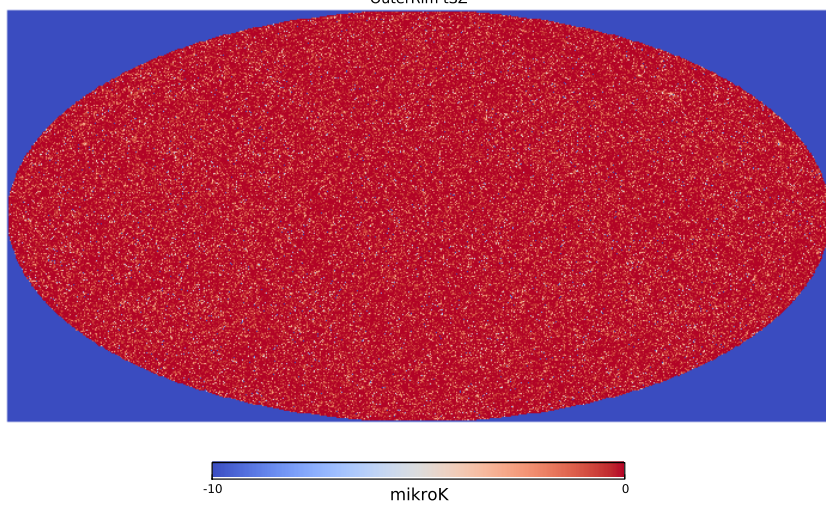

FIG. 10.- Upper panel: full-sky map of the kinematic Sunyaev-Zel'dovich effect based on the Outer Rim simulation. Lower panel: Corresponding all-sky map of the thermal Sunyaev-Zel'dovich effect.

(2015). The high mass resolution and the large volume covered by the simulations also enables us to model cosmic infrared background sources, a task that has been difficult to achieve in the past due to lack of resolution and/or volume covered. These are just a few examples for the large variety of projects that can be carried out with data from the Outer Rim simulation. The data set will be very valuable in the future to build catalogs for a range of surveys to help test pipelines and validate cosmology analysis tools.

\section{DATA RELEASE}

As part of this paper we release some of the data products publicly. A more detailed description about the data products and how to read the data is given in Heitmann et al. (2019). The data release paper (Heitmann et al. 2019) includes several more simulations besides the Outer Rim simulation. For the Outer Rim simulation we provide time snapshots of down-sampled particle files and FOF halo catalogs for the following redshifts:

$$
z=\{0.0,0.05,0.21,0.50,0.78,0.86,1.43\} \text {. }
$$

We also provide particle lightcone and halo lightcone data for one octant of a sphere. The data is stored in GenericIO format, a reader is provided that can be used to read the data into a python code or to convert the files to ASCII (the user is warned that for the downsampled particles this might not be a good idea). The downsampled particle data files are 0.4TB each, the halo catalogs vary with redshift. Each output includes metadata, describing the content of the files in detail. The halo catalogs contain information about the number of particles in each halo, a halo ID (which is by itself not meaningful), the halo mass measured in $h^{-1} \mathrm{M}_{\odot}$, the halo center given by the potential minimum, the center of mass coordinates and velocities, and the halo velocity dispersion. Centers are measured in units of $h^{-1} \mathrm{Mpc}$ and velocities are given in comoving peculiar velocities measured in $\mathrm{km} / \mathrm{s}$. The particle files contain particle positions, velocities, IDs, a potential value, and a mask. The potential value is measured on the PM grid that was used for the simulation and has not been normalized.

In order to access these data products, please visit our webpage at https://cosmology.alcf.anl.gov/, choose the Outer Rim simulation and follow the instructions given there and in Heitmann et al. (2019).

\section{CONCLUSIONS AND OUTLOOK}

In this paper we introduced the Outer Rim simulation, one of the largest cosmological simulations currently available at the volume and mass resolution covered. The simulation generated more than 5PB of raw data and we have extracted a range of useful data from the raw particle outputs for further science projects (Level 2 and Level 3 in the parlance of the paper). The detailed information about halos, their evolution over time as captured by merger trees, and the fate of their inner cores allows for the construction of sophisticated synthetic sky maps in different wavebands. We have showcased some examples here such as maps of the Sunyaev-Zel'dovich effect, but many more catalogs are currently being constructed by several science collaborations.

The simulation was carried out on Mira, a BG/Q system at the Argonne Leadership Computing Facility. In order to enable such a large simulation, our cosmology code HACC had to optimized in many ways, ranging from optimal memory usage, to fast I/O, to customization of the force kernel for the BG/Q architecture, to name just a few. We have provided some information of these optimizations in this paper with a view to proceeding to next generation machines.

Running a large simulation on basically the full machine was a major challenge, as was the analysis of the resulting data. We have carried out some of the analysis in-situ to minimize $\mathrm{I} / \mathrm{O}$ times and some of the analysis in post-processing. When carrying out in-situ analysis it is very important that the tools perform at very high efficiency, forcing the development of loadbalanced approaches that enable the most efficient use of the full machine. As part of this paper, we make some of the data products from the simulation publicly available. With time, we will make many of the synthetic catalogs based upon the simulation available as well.

Argonne National Laboratory's work was supported under the U.S. Department of Energy contract DE-AC02-06CH11357. $\mathrm{N}$. Frontiere acknowledges support from the DOE CSGF Fellowship program. This research used resources of the Argonne Leadership Computing Facility at the Argonne National Laboratory, which is supported by the Office of Science of the U.S. Department of Energy under Contract No. DE-AC0206CH11357. We are indebted to the ALCF team for their outstanding support and help to enable us to carry out a simulation at this scale. The research described in the paper was partially supported by the Scientific Discovery through Advanced Computing (SciDAC) program funded by the U.S. DOE Office of Advanced Scientific Computing Research and the Office of High Energy Physics. We thank Jon Woodring for visualization support. 


\section{REFERENCES}

Abate A. et al. [LSST DESC], arXiv:12110310 [astro-ph.CO]

Abell, P. A. et al. [LSST Science Collaborations and LSST Project Collaboration], arXiv:0912.0201 [astro-ph.IM].

Aghamousa, A., et al. [DESI Collaboration], arXiv:1611.00036 [astro-ph.IM]

Benson, A.J. 2012, New Astronomy 17, 175

Bhattacharya, S., Heitmann, K., White, M., Lukić, Z., Wagner, C., \& Habib, S. 2011, Astrophys. J. 732, 122

Bhattacharya, S., Habib, S., Heitmann, K., \& Vikhlinin, A. 2013, Astrophys. J. 766,32

Bleem, L., Stalder, B., de Haan, T., et al. 2015, ApJS, 216, 27

Child, H. L., Habib, S., Heitmann, K., Frontiere, N., Finkel, H., Pope, A., \& Morozov, V. 2018, Astrophys. J. 859, 55

Crocce, M., Fosalba, P., Castander, F. J., \& Gaztanaga, E. 2010, MNRAS 403, 1353

Davis, M. Efstathiou, G., Frenk, C.S. White, S.D.M. 1985, ApJ, 292, 371

Flender, S., Bleem, L., Finkel, H., Habib, S., Heitmann, K., \& Holder, G. 2016, Astrophys.. J. 823, 98

Gehrels, N. 2010, arXiv:1008.4936 [astro-ph.CO].

Gil-Marín, H. 2018, MNRAS 4/7, 1604

Habib, S., Pope, A., Lukić, Z., Daniel, D., Fasel, P., Desai, N., Heitmann, K., Hsu, C.-H., Ankeny, L., Mark, G., Bhattacharya, S., \& Ahrens, J. 2009, J. Phys. Conf. Ser. 180, 012019

Habib, S., Morozov, V., Finkel, H., Pope, A., Heitmann, K., Kumaran, K., Peterka, T., Insley, J., Daniel, D., Fasel, P., Frontiere, N., \& Lukić, Z. 2012, Proc. SC12, arXiv:1211.4864

Habib, S., Morozov, V., Frontiere, N., Finkel, H., Pope, A., Heitmann, K., Kumaran, K., Vishwanath, V., Peterka, T., Insley, J., Daniel, D., Fasel, P., \& Lukić, Z. 2013, Proc. SC13

Habib, S., Pope, A., Finkel, H., Frontiere, N., Heitmann, K., Daniel, D., Fasel, P., Morozov, V., Zagaris, G., Peterka, T., Vishwanath, V., Lukić, Z., Sehrish, S., \& Liao, W.-k., New Astronomy, 42, 49 (2016)

Heitmann K., Lawrence, E., Kwan, J., Habib, S., \& Higdon, D., 2014 Astrophys. J. 780, 111

Heitmann, K., Frontiere, N., Sewell, C., Habib, S., Pope, A., Finkel, H., Rizzi, S., Insley, J., \& Battacharya S. 2015, Astrophys. J. Supp., 219, 34

Heitmann, K., Bingham, D., Lawrence, E., Bergner, S., Habib, S., Higdon, D., Pope, A., Biswas, R., Finkel, H., Frontiere, N., \& Bhattacharya, S. 2016, Astrophys. J. 820, 108

Heitmann K., Uram T. et al. arXiv:1904.11966 [astro-ph.CO]

Hou, J. et al., 2018, MNRAS 480, 2521

Jenkins, A. et al. 2001, MNRAS 321, 372

Komatsu, E. et al. 2011, Astrophys. J. Supp., 192, 18

Kwan, J., Bhattacharya, S., Heitmann, K., \& Habib, S. 2013, Astrophys. J. 768, 123

Lewis, A., Challinor, A., \& Lasenby, A. 2000, Astrophys. J. 538, 473

Li, N., Gladders, M., Rangel, E., Florian, M.K., Bleem, L.E., Heitmann, K., Habib, S., \& Fasel, P. 2016, Astrophys. J. 828, 54

Li, N., Gladders, M., Heitmann, K., Rangel, Child, H., E., Florian, M.K., Bleem, L.E., Habib, S., \& Finkel, H. 2018, arXiv:1810.13330

Pope, A., Habib, S., Lukić, Z., Daniel, D., Fasel, P., Desai, N., and Heitmann, K. 2010, Comp. Sci. Eng. 12, 17

Rangel, E., Frontiere, N., Habib, S., Heitmann, K., Liao, W., Agrawal, A., \& Choudhary, A. 2017, IEEE 24th International Conference on High Performance Computing, 398

Sewell, C., Heitmann, K., Finkel, H., et al. 2015, SC'15 Proceedings of the International Conference for High Performance Computing, Networking, Storage and Analysis Article No. 50

Spergel, D. et al. 2015, arXiv:1503.03757 [astro-ph.IM]

Springel, V. et al. 2005, Nature, 435, 629

Wechsler, R. H. \& Tinker J. L. 2018, arXiv:1804.03097] [astro-ph.CO]

Woodring, J., Heitmann, K., Ahrens, J., Fasel, P., Hsu, C.-H., Habib, S., \& Pope, A. 2011, Astrophys. J. Supp. 195, 11

Zarrouk, P. et al. 2018, MNRAS 477, 1639

Zel'dovich, Y.B. 1970, A\&A, 5, 84 